\title{
Cortical GABAergic neurons: stretching it
}

\author{
Kathleen S. Rockland ${ }^{1 *}$ and Javier DeFelipe ${ }^{2}$ \\ RIKEN-MIT Center for Neural Circuit Genetics, Massachusetts Institute of Technology, Cambridge, MA, USA \\ 2 Laboratorio Cajal de Circuitos Corticales (CTB), Universidad Politécnica de Madrid and Instituto Cajal (CSIC), Madrid, Spain \\ *Correspondence: kathrock@mit.edu
}

In the cerebellum and basal ganglia, projection neurons are GABAergic; but in the cerebral cortex, there has been a historically strong dichotomy between glutamatergic projection neurons and GABAergic local circuit neurons. While this dichotomy is overwhelmingly valid, it is now clear that a small population of longdistance projecting GABAergic neurons occurs in primates, as well as in cats and rodents. Beyond their well-documented existence, however, the functional significance, ontogeny, and connectivity of this intriguing subpopulation remain obscure.

This volume brings together nine articles that are intended to provide a summary of some of the current thinking regarding cortical GABAergic neurons with long-distance connections. These cover issues of nomenclature, subtype heterogeneity, species differences, and functional importance, in the adult brain, and in development. A final article (Clancy et al.) is organized as a series of extracts and commentaries on the individual articles, each section of which is followed by general comments and discussion. The order of articles in the present volume parallels the order in this final article. The topics in the other eight articles are briefly described below.

The first article (Clancy et al.) presents a database of neural developmental events in three commonly used experimental species: rat, mouse, and macaque, using data from the online database www.translatingtime.net, as well as GABAergic and subplate developmental data from the empirical literature. Striking similarities between the two rodent populations permit the identification of developmental dates for GABAergic and subplate neural events in rats that were previously identified only in mice, and proposed timing of development events in mouse, previously identified in rats. The authors also exploit their model to produce estimates of dates for GABAergic and subplate neural events that have not yet been, or cannot be determined empirically in humans.

The next two articles focus on projections and neurochemical profiles of long-distance projecting GABAergic neurons in several cortical areas (Higo et al.) and in the hippocampal system (Jinno). The authors use a variety of techniques to visualize and characterize in detail these scattered, hard to localize subpopulations: Golgi-like filling by NADPH-d (Higo et al.), GFP-labeling of GABAergic neurons in GAD67-GFP Cre-reporter mice (Higo et al.), and combined molecular, electrophysiological, and retrograde tracer approaches (Jinno).
A cluster of three articles address the identity of white matter neurons, their role in development, and variations across species (Kanold; Luhmann et al.; Petanjek et al.). Subplate neurons are a largely transient subpopulation, morphologically, and neurochemically heterogeneous, that resides in the neonatal cortical white matter. They play a key role in the segregation and functional maturation of thalamocortical and intracortical circuits and, especially as discussed in Luhmann et al., are postulated not only to serve as a transient relay station for afferent inputs, but also as an active element amplifying the afferent and intracortical activity. Local and long-distance projections arise from subpopulations of glutamatergic and GABAergic subplate neurons. Projections to more distant neocortical regions are thought to form a corticocortical synaptic network. Clinical evidence suggests that subplate cells in the mature cortex contribute to the manifestation of abnormal neuronal circuits, pathological activity, and long-term neurological deficits.

Espinoza et al. propose a novel classification of non-subplate pioneer neurons, with distinctive neurochemical phenotypes, projections, and localization within the preplate before its partition. They report the two populations as recognizable in rat and mouse, but with species-specific neurochemical features.

Finally, Zhang et al. report a subpopulation of immature, interneuron-like cells in the association cortex and amygdala of non-human primates, and infer that these are involved in a life-long role, at least partly associated with plasticity.

In summary, these articles present a range of data and issues that are under active investigation. Even since the short time since 2009 (the original publication of these articles), technical advances have occurred that promise significant new approaches to unraveling the structure and function of this subpopulation, not only as relates to basic cortical circuitry and function but also in relation to developmental and psychiatric disorders.

Received: 17 April 2012; accepted: 05 May 2012; published online: 31 May 2012. Citation: Rockland KS and DeFelipe J (2012) Cortical GABAergic neurons: stretching it. Front. Neuroanat. 6:16. doi: 10.3389/fnana.2012.00016

Copyright $\odot 2012$ Rockland and DeFelipe. This is an open-access article distributed under the terms of the Creative Commons Attribution Non Commercial License, which permits non-commercial use, distribution, and reproduction in other forums, provided the original authors and source are credited. 